\title{
Diel and tidal variations of benthic assemblages in sediments associated with boulder fields
}

\author{
J. J. Cruz-Motta* \\ Centre for Research on Ecological Impacts of Coastal Cities, Marine Ecology Laboratories, A11, University of Sydney, \\ New South Wales 2006, Australia
}

Present address: Depto. Estudios Ambientales, Universidad Simón Bolívar, Apado Post 89000, Sartenejas, Caracas 1080-A, Venezuela

\begin{abstract}
During daytime low tides, assemblages living in sediments below boulders differ from those living in sediments not overlaid by boulders. It is, however, not known whether these differences are consistent under different tidal or diel conditions. In this study, the composition of assemblages living in sediments associated with boulder fields (below and adjacent to boulders) under different diel (day and night) and tidal (high and low) conditions, was investigated. Significant differences between assemblages living in sediments below boulders and those living in sediments without boulders persisted during different combinations of daylight and state of tide. Species responsible for these differences did, however, change in relation to the level of the tide, but not to time of day. The presence of boulders in sediments allows the coexistence of species usually found on rocky substrata with those usually found in sediments. Mechanisms to explain intertidal movements of invertebrates in either hard or soft-bottom benthic assemblages have been very well described in the literature. The patterns of spatial distribution, in relation to diel and tidal fluctuations, of individual taxa found in this study, however, cannot easily be explained by these mechanisms. Consequently, the mechanisms of migrational movements of benthic assemblages associated with boulder fields need development of new conceptual models. A model considering the structural complexity of the boulder fields and the interactions between hard- and soft-bottom habitats is proposed herein.
\end{abstract}

KEY WORDS: Tidal movements · Diel movements · Intertidal boulder fields · Macrobenthos · Intertidal migration

Resale or republication not permitted without written consent of the publisher

\section{INTRODUCTION}

The effects of diel and tidal variations on many natural biological and physical processes have been long and widely recognised. More specifically, organisms in coastal areas show strong ecological (e.g. Cabral \& Costa 2001, Saigusa 2001), physiological (e.g. Yamaguchi 2001, Gevaert et al. 2003) and/or behavioural (e.g. Kim \& Choe 2003) responses to the cycles of the solar day and/or tides. These responses may be controlled by exogenous factors, as reported for the mussel Mytilus edulis (Ameyaw-Akmufi \& Naylor 1987) and/or endogenous factors, as reported for the crab Carcinus maenas (Naylor 1988). One of these responses is migration, which has conspicuous and easily observable consequences to the patterns of spatial distribution of different components of an assemblage related to the time of the day and the level of the tide. Most studies on the effects of diel and tidal variations on migration have been on individual species, including representatives of almost every major group of organisms, ranging from microphytobenthos (e.g. Blanchard et al. 2002) to dolphins (e.g. Mendes et al. 2002) . Very few studies have, however, looked at more than one species simultaneously in the same habitat (e.g. Hicks 1992, Saigusa \& Kazushi 2000).

In addition, many studies of invertebrates have been done on rocky platforms, where movements generally occur in a 'horizontal' way, either up and down the shore or in and out of refuges in accordance with the phase of solar day or tide (e.g. Chelazzi et al. 1988). Alternatively, studies have been done on intertidal sand/mud flats, where movements generally have a 'vertical' component (Yannicelli et al. 2001); i.e. 
animals emerge from the sediment into the water column, where they may move 'horizontally' up and down the shore (e.g. Armonies 1992, Takahashi \& Kagawuchi 1997), and return to the sediment in accordance with the solar or tidal cycle. On the other hand, the study of intertidal migration has been ignored in complex systems such as boulder fields, which contain hard and soft substrata. Boulders lying on soft sediments create a series of particular micro-environments (the top, the undersurface and the substrata below the boulder) that not only house species unique to this habitat (Cruz-Motta et al. 2003), but also allow the coexistence of species living on rocky substrata and in sediments (Chapman 2002). The particular assemblages living in boulder fields are likely to migrate in response to diel and/or tidal fluctuations, which affect patterns of spatial distribution. Moreover, mechanisms of intertidal migration in boulder fields could comprise combinations of mechanisms already known for either hard or soft substrata.

Assemblages in sediments below boulders are different from assemblages in sediments away from boulders (Cruz-Motta et al. 2003). The results of CruzMotta et al. (2003) were variable, but some taxa such as Bittium granarium (Gastropoda, Cerithiidae), Amphipholes sp. (Echinodermata Ophiuroida) and Cirriformia sp. (Cirratulidae, Polychaeta), among others, were only present below boulders. Given that the study by Cruz-Motta et al. (2003) was done during daytime low tide, it is important to discover whether these differences are consistent under different diel or tidal conditions, since further development of models and associated hypotheses about processes affecting assemblages in these microhabitats will greatly depend on such knowledge.

It is therefore necessary to establish whether (1) assemblages characteristically found below or away from boulders during low tide during the day are specific to these micro-environments, irrespective of diel and tidal conditions (i.e. they comprise a permanent habitat), or (2) animals use the various microenvironments only during certain diel and/or tidal conditions (i.e. they are temporary habitats). In order to evaluate these alternative models, the present study describes patterns of spatial distribution of benthic assemblages living in sediments associated with boulder fields under different combinations of times of the day (day versus night) and tidal levels (high versus low). Under the 'permanent habitat' model, it was predicted that differences between assemblages living in sediments below boulders and those in sediments away from boulders would be consistent under any diel/tidal combination. Consistent means that the magnitude of the average dissimilarities between assemblages below and those away from boulders and that the species responsible for those differences will not differ among different combinations of diel and tidal conditions. Under the 'temporary habitat' model, at least 1 of the above predictions will not be true. If this second model is supported, it is implicit that differences are due to diel and/or tidal migrations of some or all components of the assemblages living in sediments associated with boulder fields.

Although very specific mechanisms have been proposed for diel and tidal migrations on rocky or in soft substrata, there is no information on diel and tidal migrations of macrobenthonic organisms in boulder fields. Consequently, in addition to testing the above hypotheses, this study describes patterns of spatial distribution of individual taxa in relation to microhabitat (below vs away from boulders) and diel and tidal conditions in order to propose a conceptual model about specific patterns of movements that may be peculiar to boulder fields.

\section{MATERIALS AND METHODS}

Study sites and sampling. Intertidal boulder fields at 3 different locations (Chowder Bay, Hermit Point and Watson's Bay) in Sydney Harbour (New South Wales, Australia) were sampled (Fig. 1). These boulder fields share similar characteristics; they are between 50 to $80 \mathrm{~m}$ in length, adjacent to reflective beaches in narrow sheltered bays inside the harbour, and protected from oceanic swells, resulting in little wave-action during most of the year (Short \& Trenaman 1992, Short 1993). Boulders at each location are mainly composed of sandstone and the sizes considered in this study $\left(\approx 1000 \mathrm{~cm}^{2}\right.$ undersurface area) are minimally disturbed by normal wave-conditions (McGuinness 1984). Boulders typically lie on a layer of sediment, covering a rock platform. The depth of the sediment layer varies between 5 to $12 \mathrm{~cm}$ across the 3 locations. Sediments under boulders are typically 'fine sands', with a minimal $(<1 \%)$ content of fine material $(<0.063 \mathrm{~mm})$, with the exception of Watson's Bay that has a coarser sediment ('medium sands').

Each location was sampled under different diel (day and night) and tidal (high and low) conditions on 3 different, randomly selected occasions (i.e. there were 3 replicate times of sampling for each condition; Fig. 2) between October and November 2002. Low or high tides were selected at noon or midnight $( \pm 2.5 \mathrm{~h})$ during spring tides only, to avoid confounding any potential effects of 2 cycles (solar day and tides) with different periods and synodic variations (29 d).

At each combination of location, diel and tidal condition and time, 6 haphazardly chosen boulders of similar size (approximately $1000 \mathrm{~cm}^{2}$ ) were totally (low 


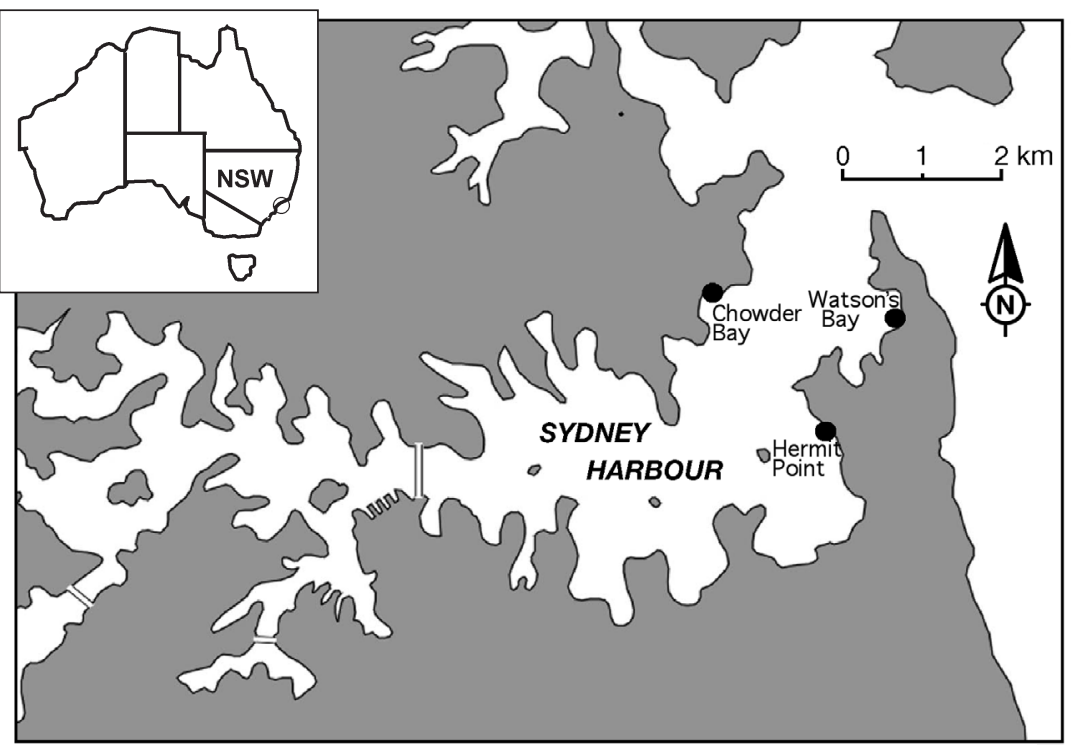

Fig. 1. Study sites in the metropolitan area of Sydney, NSW, Australia seawater and stained with neutral red. Samples were later sorted and animals identified to the lowest taxonomic level possible (mainly families and genera). Within each family or genera, animals were distinguished as morpho-species (Oliver \& Beattie 1993). Identification to formal species level was difficult to achieve because many of the higher taxa in Australia have not been welldescribed or have recently been revised. Despite a considerable increase in efforts to describe estuarine invertebrate species in Australia in recent years, a lot of work still needs to be done (Hutchings 1999). Furthermore, some of the animals found in this study would have required dissecting to determine the species, and this was impractical given the number of samples collected.

tide) or partially (high tide) overturned and the sediment below sampled using an $18 \mathrm{~cm}$ diameter corer, which was pushed down until it made contact with the rock platform. Sediments in the cores were retrieved using an electrically powered suction-sampler. Once sampled, boulders were returned to their original positions. We sampled 6 randomly chosen areas of sediment of equivalent size $\left(1000 \mathrm{~cm}^{2}\right)$, at least $0.5 \mathrm{~m}$ away from each boulder in the same way. The maximum distance between any 2 boulders at these sites ranges between 1 and $1.5 \mathrm{~m}$, so $0.5 \mathrm{~m}$ represents a realistic distance away from a boulder.

Samples were taken within a band 1.5 to $2 \mathrm{~m}$ upshore of the lower intertidal edge of the boulder field. Samples were sieved using a $1 \mathrm{~mm}$ sieve, stored in a bag, fixed with a buffered $7 \%$ solution of formalin in

Locations:
Level of tide:
Times:
Microhabe of day:

Fig. 2. Experimental design. Below: beneath boulder; Away from: sediment $\geq 0.5 \mathrm{~m}$ distant from relevant boulder; further details in 'Materials and methods'
Analyses of data. Bray-Curtis similarity matrices of samples were constructed from the original biological data matrices. Non-metric multidimensional scaling (nMDS) was used to illustrate patterns in the spatial distribution of the samples. Typically, centroids were plotted; i.e. Bray-Curtis dissimilarity measures were calculated for the averages of the 6 replicates across each one of the treatment combinations. Analyses of similarities (ANOSIM in PRIMER [Plymouth routines in multivariate ecological analysis]; Clarke 1993) tested the null hypothesis of no differences between assemblages in sediments below boulders and assemblages in sediments away from boulders, for each possible combination of locations, diel and tidal conditions and times. When significant differences were found, the taxa making the greatest contribution to these differences were detected using similarity percentages (SIMPER analysis) (PRIMER; Clarke 1993).

To test the null hypothesis of no differences in the magnitude of dissimilarities (between assemblages in sediments below and away from boulders) under different combinations of diel and tidal conditions, 5-factor analyses of variance (ANOVA) tested for differences in this variable among locations (random), tide (fixed and orthogonal), time of day (fixed and orthogonal) and treatment (below and away from boulders, fixed and orthogonal), at 3 different times (random and nested within the first 3 factors). Independent dissimilarity measures were obtained by randomly selecting pairs of samples from the 2 groups of 6 replicate samples (below and away from boulders) per each combination of location, diel and tidal condition and time, in such a way that independent replicate measures of dissimilarity (2) were obtained for (1) dissimilarities within 
samples taken below boulders, (2) dissimilarities within samples taken away from boulders and (3) dissimilarities between treatment groups (below vs away from boulders). Additionally, similar analyses of variance (5-factor) were done on the abundance of some taxa (those identified in the SIMPER analyses). Heterogeneity of variances were tested using Cochran's $C$-test, and transformation ( $\ln x+1)$ was done when appropriate, following the recommendations of Underwood (1997) and McGuinness (2002).

\section{RESULTS}

In a total of 432 samples, there were 157 taxa, of which between 10 and 21 taxa comprised $75 \%$ of all animals at each location (Table 1). Of these abundant taxa, only Ericthonius sp. (Gammarida), Amphipholes sp. (Ophiuroidea) and Birubius sp. (Gammarida) were common to all 3 locations.

As previously observed during daytime low tides, assemblages from below boulders were clearly different from those away from boulders in any diel/tidal combination (Fig. 3). These patterns were mostly confirmed by analyses of dissimilarities within and between treatments (i.e. below and away from boulders; Table 2). Despite the significant third-order interaction

Table 1. Most abundant taxa (totalling $75 \%$ of total abundance) in assemblages sampled at 3 locations, Sydney, Australia. N: no. of taxa; n: no. of individuals

\begin{tabular}{|c|c|c|c|}
\hline Class & $\begin{array}{l}\text { Chowder Bay } \\
(\mathrm{N}=120 ; \mathrm{n}=8421)\end{array}$ & $\begin{array}{l}\text { Hermit Point } \\
(\mathrm{N}=114 ; \mathrm{n}=4161)\end{array}$ & $\begin{array}{l}\text { Watson's Bay } \\
(\mathrm{N}=114 ; \mathrm{n}=4680)\end{array}$ \\
\hline Cnidaria & & & Actinia tenebrosa \\
\hline Crustacea & $\begin{array}{l}\text { Anthuridae sp. B } \\
\text { Dexaminidae sp. A } \\
\text { Ericthonius sp. } \\
\text { Hyale sp. } \\
\text { Melita sp. } \\
\text { Mysidacea sp. A } \\
\text { Birubius sp. } \\
\text { Podoceridae sp. A } \\
\text { Tannaidacea sp. B }\end{array}$ & $\begin{array}{l}\text { Anthuridae sp. B } \\
\text { Dexaminidae sp. C } \\
\text { Ericthonius sp. } \\
\text { Paraphoxus sp. } \\
\text { Gammaridae sp. D } \\
\text { Hyale sp. } \\
\text { Leucothoidae sp. A } \\
\text { Melita sp. } \\
\text { Birubius sp. } \\
\text { Sphaeromatidae sp. A }\end{array}$ & $\begin{array}{l}\text { Aora sp. } \\
\text { Ericthonius sp. } \\
\text { Isaeidae sp. A } \\
\text { Pagurus sp. } \\
\text { Birubius sp. }\end{array}$ \\
\hline Gastropoda & & Bittium granarium & Bittium granarium \\
\hline Nemertini & & & Nemertean sp. A \\
\hline Oligochaeta & & Oligochaete sp. A & \\
\hline Polychaeta & & $\begin{array}{l}\text { Capitella sp. } \\
\text { Mediomastus sp. } \\
\text { Cirriformia sp. } \\
\text { Nereididae sp. B } \\
\text { Orbiniidae sp. A } \\
\text { Syllidae sp. A }\end{array}$ & $\begin{array}{l}\text { Capitella sp. } \\
\text { Cirriformia sp. } \\
\text { Eunice sp.p. B } \\
\text { Nereididae sp. B } \\
\text { Orbiniidae A } \\
\text { Syllidae sp. A }\end{array}$ \\
\hline Sipunculida & & $\begin{array}{l}\text { Golfingiidae sp. A } \\
\text { Phascolosoma sp. }\end{array}$ & $\begin{array}{l}\text { Golfingiidae sp. A } \\
\text { Phascolosoma sp. }\end{array}$ \\
\hline Holothuria & Amphipoles sp. & Amphipoles sp. & $\begin{array}{l}\text { Amphipoles sp. } \\
\text { Chiridota cf. gigas }\end{array}$ \\
\hline
\end{tabular}
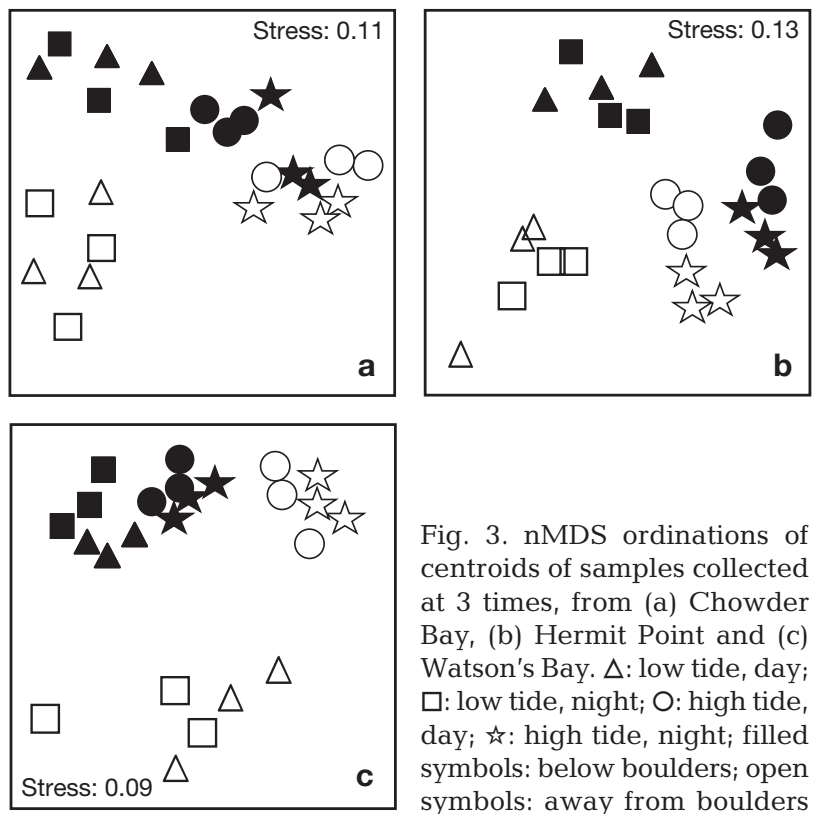

Fig. 3. nMDS ordinations of centroids of samples collected at 3 times, from (a) Chowder Bay, (b) Hermit Point and (c) Watson's Bay. $\triangle$ : low tide, day; $\square$ : low tide, night; O: high tide, day; is: high tide, night; filled symbols: below boulders; open symbols: away from boulders

among location, tide and treatment, a posteriori comparisons (Student-Newman-Keuls tests) showed that dissimilarities between assemblages below and those away from boulders were larger than dissimilarities among replicates in samples taken below boulders (in 5 of 6 possible combinations) and among replicates in samples taken away from boulders (always) (Table 2, Fig. 4). As there was no significant variability among 'times' in analyses of dissimilarities (Table 3), times were averaged (Fig. 4).

In addition, Fig. 3 shows that assemblages sampled during low tide were clearly separated from assemblages sampled during high tide; this was particularly conspicuous at Hermit Point (Fig. 3b). Similarly, the magnitude of the average dissimilarity between assemblages found below boulders and those away from boulders was significantly different in relation to tide across all 3 locations (Table 2, Fig. 4). These results support the temporary habitat model that assemblages use different microenvironments in sediments associated with boulder fields only during certain diel and tidal conditions. More specifically, it would appear that only tidal variations are important. No significant effect of day/night was found in the structure of assemblages (Table 3, Fig. 3). 
Table 2. A posteriori comparisons (Student-Newman-Keuls tests) among combinations of treatments of the significant third-order interaction (location $\times$ tide $\times$ treatment: see Table 3). BE: within samples from sediments below boulders; OUT: within samples from sediments away from boulders; BET: between samples collected in these environments. $>$ and $<$ indicate significant differences at $p<0.05$

\begin{tabular}{|c|c|c|c|c|}
\hline \multicolumn{2}{|l|}{ Source } & \multirow{2}{*}{$\begin{array}{l}\text { Chowder Bay } \\
\text { BET }=\text { BE > OUT }\end{array}$} & \multirow{2}{*}{$\begin{array}{l}\text { Hermit Point } \\
\text { BET }>\text { BE }=\text { OUT }\end{array}$} & \multirow{2}{*}{$\begin{array}{l}\text { Watson's Bay } \\
\text { BET > BE = OUT }\end{array}$} \\
\hline Within and & Low tide & & & \\
\hline between samples & High tide & $\mathrm{BET}>\mathrm{BE}=\mathrm{OUT}$ & $\mathrm{BET}>\mathrm{BE}>\mathrm{OUT}$ & $\mathrm{BET}>\mathrm{BE}=\mathrm{OUT}$ \\
\hline \multirow[t]{3}{*}{ Tidal comparison } & $\mathrm{BE}$ & Low $>$ High & Low > High & Low = High \\
\hline & OUT & Low $>$ High & Low $=$ High & Low > High \\
\hline & BET & Low $>$ High & Low $>$ High & Low $>$ High \\
\hline
\end{tabular}

Moreover, the taxa responsible for $70 \%$ of the total differences between assemblages in sediments below boulders and those away from boulders mainly varied with tidal condition and between locations (Table 4). During the day or night, only between 6 and $21 \%$ of taxa (depending on the location), were found at low and high tide. Conversely, during low or high tide, between 25 and $60 \%$ of taxa responsible for differences between assemblages below and those outside boulders, were found during the day and night.

Analyses of individual taxa revealed the existence of 11 different patterns of spatial distribution in relation to the microhabitat (below vs away from boulders), diel and tidal condition (Table 5, Fig. 5): (1) taxa found below boulders almost exclusively during low tide (e.g. Fig. 5a); (2) taxa found in sediments away from boul-

Table 3. Analysis of variance of 3 types of dissimilarity (treatments [Tr]: BE, OUT and BET; see Table 2) at 3 random locations (L), 2 fixed tidal $(\mathrm{H})$ levels (low and high) and 2 fixed diel (D) conditions (day and night) during 3 random times $(T i$, nested in Location $\times$ Tide $\times$ Diel $)$. Significance: ${ }^{*} \mathrm{p}<0.05$; ${ }^{* *} \mathrm{p}<0.01 ; \mathrm{ns}=$ not significant

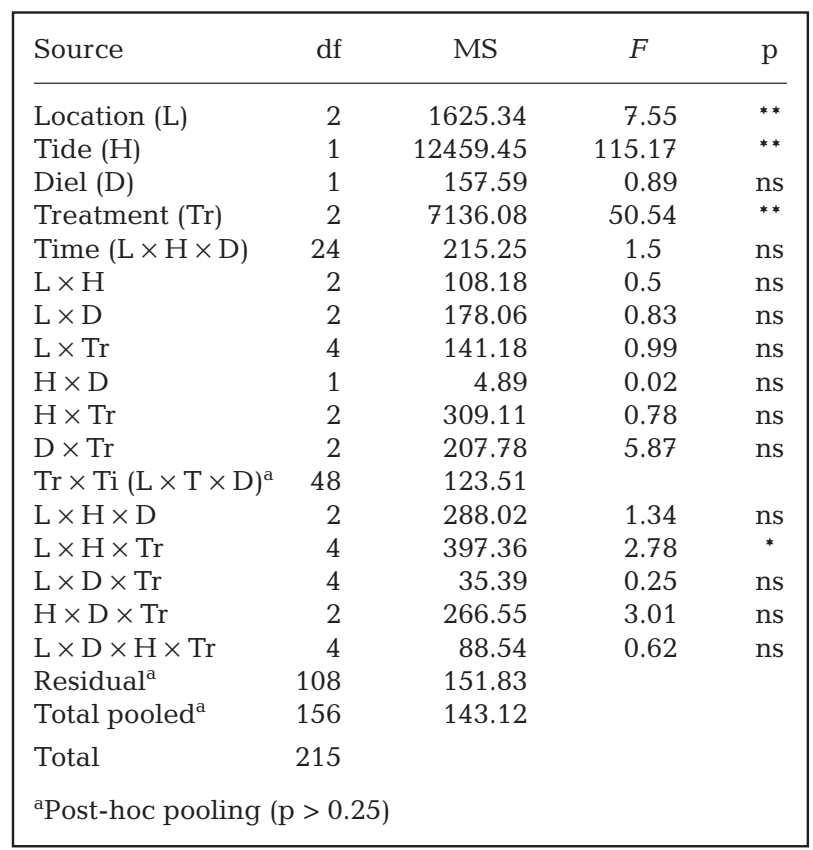
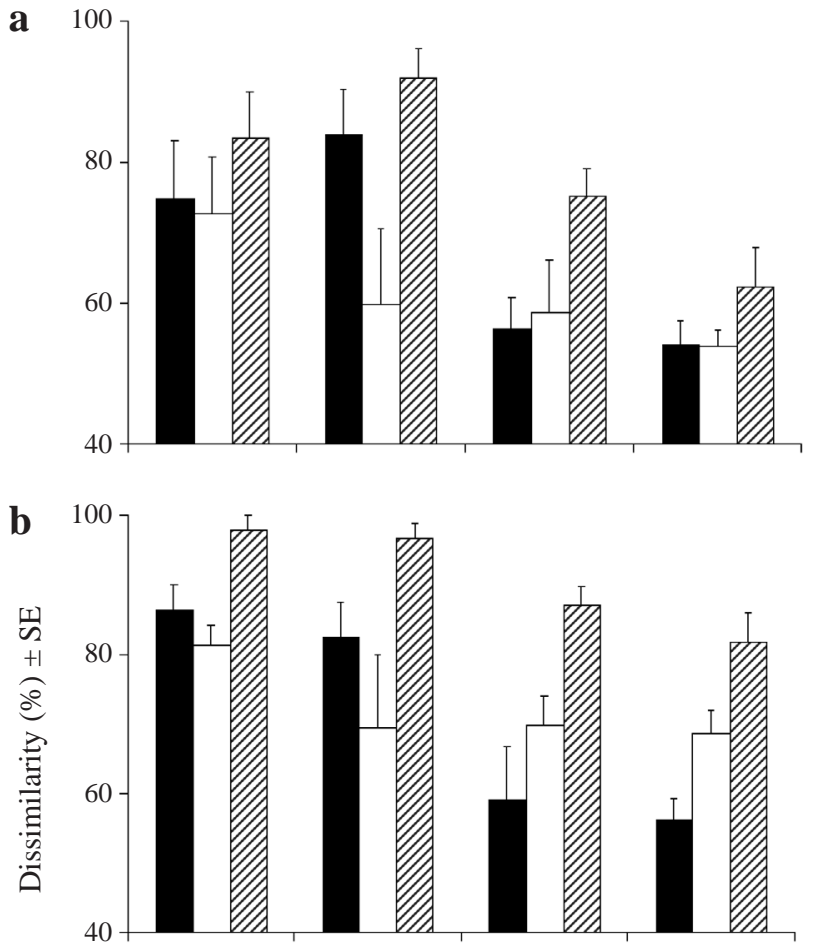

c

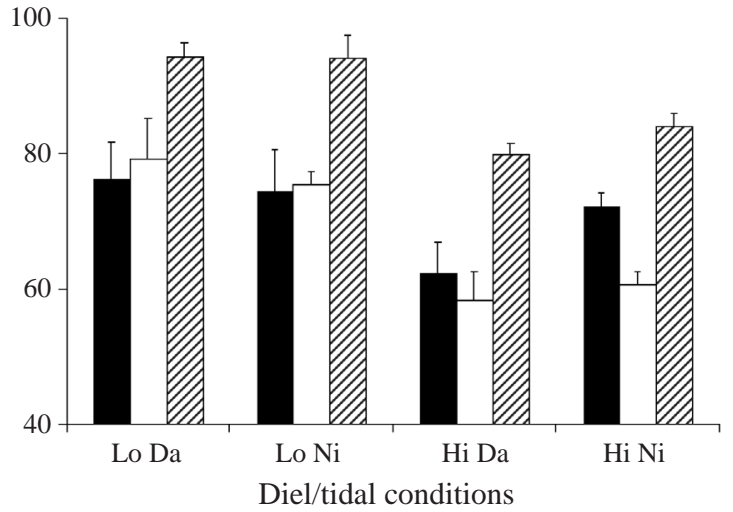

Fig. 4. Bray-Curtis dissimilarity values for 4 different diel/tidal combinations - low day (Lo Da), low night (Lo Ni), high day (Hi Da) and high night (Hi Ni) - at 3 locations - (a) Chowder Bay, (b) Hermit Point and (c) Watson's Bay. ש: dissimilarities within samples collected in sediments below boulders; $\square$ : dissimilarities within samples collected in sediments away from boulders; $\nabla$ : dissimilarities between the 2 groups of samples (below and away from boulders) 
Table 4. Taxa responsible for $70 \%$ of total dissimilarity (SIMPER) between assemblages sampled in sediments below boulders and sediments away from boulders at 3 locations and 4 diel/tidal combinations. Percentages of taxa common to the 3 locations under each of the diel/tidal combinations are also indicated

\begin{tabular}{|c|c|c|c|c|}
\hline Condition & Chowder Bay & Hermit Point & Watson's Bay & \% common \\
\hline Low tide, day & $\begin{array}{l}\text { Hyale sp. } \\
\text { Mediomastus sp. } \\
\text { Phoxocephalidae sp. A } \\
\text { Amphipholes sp. } \\
\text { Melita sp. } \\
\text { Cirriformia sp. }\end{array}$ & $\begin{array}{l}\text { Hyale sp. } \\
\text { Cirolanidae sp. A } \\
\text { Golfingiidaer sp. A } \\
\text { Oligochaeta sp. A } \\
\text { Mediomastus sp. } \\
\text { Syllidae sp. A }\end{array}$ & $\begin{array}{l}\text { Nemertean sp. A } \\
\text { Syllidae sp. A } \\
\text { Birubius sp. } \\
\text { Actinia tenebrosa } \\
\text { Cirriformia sp. } \\
\text { Bittium granarium } \\
\text { Ericthonius sp. }\end{array}$ & 0 \\
\hline Low tide, night & $\begin{array}{l}\text { Hyale sp. } \\
\text { Birubius sp. } \\
\text { Mediomastus sp. } \\
\text { Ericthonius sp. }\end{array}$ & $\begin{array}{l}\text { Hyale sp. } \\
\text { Cirolanidae sp. A } \\
\text { Cirriformia sp. } \\
\text { Paraphoxus sp. } \\
\text { Ericthonius sp. } \\
\text { Patiriella exigua } \\
\text { Anthuridae sp. B } \\
\text { Mediomastus sp. } \\
\text { Birubius sp. }\end{array}$ & $\begin{array}{l}\text { Nemertean sp. A } \\
\text { Sphaeromatidae sp. A } \\
\text { Birubius sp. } \\
\text { Bittium granarium } \\
\text { Amphipholes sp. } \\
\text { Mediomastus sp. } \\
\text { Syllidae sp. A } \\
\text { Cirriformia sp. } \\
\text { Paraphoxus sp. }\end{array}$ & 10 \\
\hline High tide, day & $\begin{array}{l}\text { Melita sp. } \\
\text { Anthuridae sp. B } \\
\text { Ericthonius sp. } \\
\text { Aora sp. }\end{array}$ & $\begin{array}{l}\text { Bittium granarium } \\
\text { Paraphoxus sp. } \\
\text { Birubius sp. } \\
\text { Melita sp. } \\
\text { Amphipholes sp. } \\
\text { Capitella sp. } \\
\text { Orbiniidae sp. A } \\
\text { Syllidae sp. A }\end{array}$ & $\begin{array}{l}\text { Syllidae sp. A } \\
\text { Isaeidae sp. A } \\
\text { Ericthonius sp. } \\
\text { Aora sp. } \\
\text { Pagurus sp. } \\
\text { Amphipholes sp. } \\
\text { Chiridota cf. gigas } \\
\text { Birubius sp. } \\
\text { Orbiniidae sp. A }\end{array}$ & 7 \\
\hline High tide, night & $\begin{array}{l}\text { Dexaminidae sp. A } \\
\text { Anthuridae sp. B } \\
\text { Tannaidacea sp. B } \\
\text { Ericthonius sp. }\end{array}$ & $\begin{array}{l}\text { Bittium granarium } \\
\text { Ericthonius sp. } \\
\text { Dexaminidae sp. C } \\
\text { Leucothoidae sp. A } \\
\text { Birubius sp. } \\
\text { Melita sp. } \\
\text { Capitella sp. }\end{array}$ & $\begin{array}{l}\text { Isaeidae sp. A } \\
\text { Ericthonius sp. } \\
\text { Actinia tenebrosa } \\
\text { Capitella sp. } \\
\text { Aora sp. } \\
\text { Syllidae sp. A } \\
\text { Chiridota cf. gigas } \\
\text { Nereididae sp. B } \\
\text { Pagurus sp. }\end{array}$ & 6 \\
\hline
\end{tabular}

ders almost exclusively during low tide (e.g. Fig. 5b); (3) taxa found in sediments below boulders almost exclusively during high tide (e.g. Fig. 5c); (4) taxa found in sediments away from boulders almost exclusively during high tide (e.g. Fig. 5d); (5) taxa that were more abundant during high than low tide, but in similar numbers in sediments below or away from boulders (e.g. Fig. 5e); (6) taxa found in sediments below boulders almost exclusively during low tide but which, during high tide, were more abundant in sediments away from than below boulders (e.g. Fig. 5f); (7) taxa found almost exclusively below boulders, but in larger numbers during the day than the night (e.g. Fig. 5g); (8) taxa found only during high tides at night in similar numbers in sediments below or away from boulders (e.g. Fig. 5h); (9) taxa found almost exclusively in sediments below boulders under any diel and tidal combination (e.g. Fig. 5i); (10) taxa found almost exclusively in sediments away from boulders under any diel/tidal conditions (e.g. Fig. 5j) and (11) taxa showing no clear pattern of spatial distribution, in most cases due to great temporal variability (Table 5).

As for the entire assemblage, most of these individual taxa showed patterns of spatial distribution that could be explained by the temporary habitat model (Patterns 1 to 8; Table 5, Fig. 5a-h); i.e. they were found in a particular microhabitat associated with the boulder field only during certain diel and tidal combinations. Nevertheless, a few taxa showed patterns of spatial distribution that could be explained by the permanent habitat model. They were always found in the same microhabitat, either below (Pattern 9; Table 5, Fig. 5i) or away from (Pattern 10; Table 5, Fig. 5j) boulders, during any diel and/or tidal condition.

Most taxa with patterns of spatial distribution that could be explained by the temporary habitat model, showed a strong pattern associated with tidal fluctuations (Patterns 1 to 6; Table 5, Fig. 5a-f) and only a few showed a pattern associated with diel or tidal and diel changes (Patterns 7 and 8; Table 5, Fig. 5g,h). 
Table 5. Patterns of spatial distribution of most important taxa. Low: low tide; high: high tide; below: sediments below boulders; away from: sediments away from boulders; day: during day; night: during night; always: under any combination of diel and tidal condition

\begin{tabular}{|c|c|c|c|c|}
\hline Pattern & Description & Chowder Bay & Hermit Point & Watson's Bay \\
\hline 1 & $\begin{array}{l}\text { Low }>\text { high } \\
\text { Below }>\text { away from }\end{array}$ & Mediomastus sp. & Patiriella exigua & Paraphoxus sp. \\
\hline 2 & $\begin{array}{l}\text { Low }>\text { high } \\
\text { Away from }>\text { below }\end{array}$ & Hyale sp. & $\begin{array}{l}\text { Cirolanidae B } \\
\text { Hyale sp. }\end{array}$ & Sphaeromatidae sp. A \\
\hline 3 & $\begin{array}{l}\text { High }>\text { low } \\
\text { Below }>\text { away from }\end{array}$ & $\begin{array}{l}\text { Bittium granarium } \\
\text { Orbiniidae sp. A } \\
\text { Amphipholes sp. }{ }^{\mathrm{a}}\end{array}$ & $\begin{array}{l}\text { Bittium granarium } \\
\text { Paraphoxus sp. } \\
\text { Birubius sp. } \\
\text { Amphipholes sp. } \\
\text { Orbiniidae sp. A }\end{array}$ & $\begin{array}{l}\text { Pagurus sp. } \\
\text { Golfingiidae sp. A } \\
\text { Idoteidae sp. A } \\
\text { Orbinnidae sp. A }{ }^{\text {b }}\end{array}$ \\
\hline 4 & $\begin{array}{l}\text { High }>\text { low } \\
\text { Away from }>\text { below }\end{array}$ & $\begin{array}{l}\text { Anthuridae sp. B } \\
\text { Erichtonius sp. } \\
\text { Tannaidacea sp. B } \\
\text { Aora sp. } \\
\text { Anthuridae sp. A }\end{array}$ & $\begin{array}{l}\text { Pyramidellidae A } \\
\text { Cilindrobelerididae sp. A } \\
\text { Soletellina sp. }\end{array}$ & $\begin{array}{l}\text { Capitella sp. } \\
\text { Aora sp. } \\
\text { Eunice sp. } \\
\text { Isaeidae sp. A } \\
\text { Erichtonius sp. }\end{array}$ \\
\hline 5 & $\begin{array}{l}\text { High }>\text { low } \\
\text { Below = away from }\end{array}$ & $\begin{array}{l}\text { Podoceridae sp. A } \\
\text { Hymenosomoatidae sp. A }\end{array}$ & $\begin{array}{l}\text { Capitella sp. } \\
\text { Melita sp. }\end{array}$ & Nereididae sp. B \\
\hline 6 & $\begin{array}{l}\text { At high tide: away from > below } \\
\text { At low tide: below > away from }\end{array}$ & Hemichordata sp. A & Oligochaeta sp. A & $\begin{array}{l}\text { Actinia tenebrosa } \\
\text { Nematonereis sp. }\end{array}$ \\
\hline 7 & $\begin{array}{l}\text { Day }>\text { night } \\
\text { Below }>\text { away from }\end{array}$ & $\begin{array}{l}\text { Pilumnidae sp. A } \\
\text { Alphidae sp. A }\end{array}$ & Syllidae sp. A & Syllidae sp. A \\
\hline 8 & $\begin{array}{l}\text { Only during high tide at night } \\
\text { Below = away from }\end{array}$ & Mysidacea sp. A & $\begin{array}{l}\text { Dexaminidae sp. C } \\
\text { Erichtonius sp. } \\
\text { Leucothidae sp. A }\end{array}$ & \\
\hline 9 & $\begin{array}{l}\text { Below > away from } \\
\text { Always }\end{array}$ & $\begin{array}{l}\text { Phascolosoma sp. } \\
\text { Polyonidae sp. A }\end{array}$ & $\begin{array}{l}\text { Cirriformia sp. } \\
\text { Golfingiidae sp. A } \\
\text { Phascolosoma sp. }\end{array}$ & $\begin{array}{l}\text { Bittium granarium } \\
\text { Cirriformia sp. } \\
\text { Birubius sp. } \\
\text { Phascolosoma sp. }\end{array}$ \\
\hline 10 & $\begin{array}{l}\text { Away from > below } \\
\text { Always }\end{array}$ & Emerita sp. & Hemichordata sp. A & \\
\hline No & $\begin{array}{l}\text { No pattern, } \\
\text { high temporal variability }\end{array}$ & $\begin{array}{l}\text { Birubius sp. } \\
\text { Melita sp. } \\
\text { Cirriformia sp. }\end{array}$ & $\begin{array}{l}\text { Anthuridae sp. B } \\
\text { Mediomastus sp. } \\
\text { Nereididae sp. B }\end{array}$ & $\begin{array}{l}\text { Amphipholes sp. } \\
\text { Nemertean sp. A } \\
\text { Mediomastus sp. }\end{array}$ \\
\hline
\end{tabular}

\section{DISCUSSION}

At each location and each time sampled, assemblages living in sediments below boulders were different from assemblages living in sediments away from boulders under any diel and tidal combination. Never-

Table 6. Percentage of taxa in Table 5, shared between day and night under each tidal condition and between low and high tides at each diel condition at the 3 locations studied

\begin{tabular}{|llccc|}
\hline Condition & $\begin{array}{c}\text { Chowder } \\
\text { Bay }\end{array}$ & $\begin{array}{c}\text { Hermit } \\
\text { Point }\end{array}$ & $\begin{array}{c}\text { Watson's } \\
\text { Bay }\end{array}$ \\
\hline Day vs & Low & 43 & 25 & 46 \\
Night & High & 60 & 38 & 46 \\
Low vs & Day & 13 & 8 & 21 \\
High & Night & 14 & 20 & 6 \\
\hline
\end{tabular}

theless, the species responsible for these differences varied markedly with level of the tide, but not with time of day (Table 6). These results support the temporary habitat model, implying that different microhabitats associated with sediments in boulder fields (below and away from boulders) are used by most invertebrate species during different phases of the tide. There was a clear temporal separation of the use of the resources provided by boulders (e.g. refuge, shelter, food, mating ground, etc.), associated with level of the tide.

Although these patterns were similar for different locations, species composition differed between locations, indicating that different species were subjected to similar ecological processes at different locations. In contrast, in some cases, the same taxon showed different patterns of spatial distribution in relation to time of day and level of tide in different locations. For example, Bittium granarium at Watson's Bay was always more 

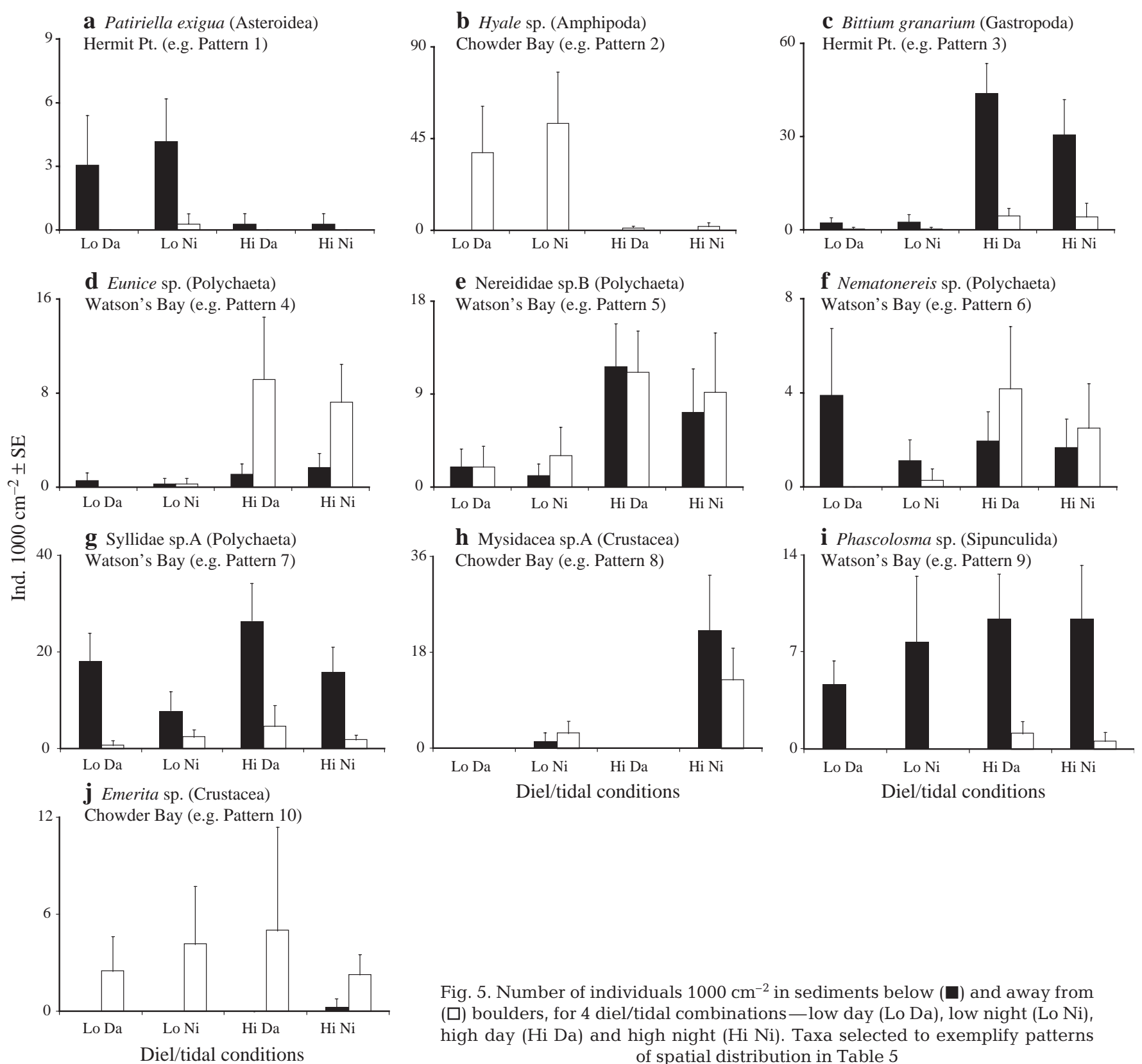

Fig. 5. Number of individuals $1000 \mathrm{~cm}^{-2}$ in sediments below (ם) and away from ( $\square$ ) boulders, for 4 diel/tidal combinations - low day (Lo Da), low night (Lo Ni), high day (Hi Da) and high night (Hi Ni). Taxa selected to exemplify patterns of spatial distribution in Table 5

abundant in sediment below boulders than in sediments away from boulders in any diel and tidal combination, whereas at Hermit Point and Chowder Bay it was also more abundant in sediments below boulders than in sediments away from boulders, but only during high tide. This species was almost absent from samples taken during low tide. More conspicuously, the unidentified Gammaridae sp. A, was always found below boulders at Watson's bay during low tide, while at Hermit Point it was below boulders during high tide.

Other studies have reported a site-specific behaviour. For example, the coastal European sea urchin Paracentrotus lividus migrates up and down the shore only dur- ing the day in some areas of the Mediterranean, in contrast to other studies in other areas where this sea urchin only migrates up and down shore during the night (Barnes \& Crook 2001). These differences between locations in patterns shown by individuals of the same species may be due to differences in physical and/or chemical conditions, such as the composition and/or structure of the sediment (Saigusa 2001), or to differences in population structure at different locations. For example, it has been reported that juveniles of the crab Carcinus maenas, the isopod Eurydice pulcra and the amphipod Orchestoidea tuberculata behave differently from the adults (Naylor \& Kennedy 2003). 
As found for the entire assemblage, univariate analyses demonstrated significant differences in relative abundances of most of the taxa in relation to diel and/or tidal fluctuations, supporting the temporary habitat model. Of all the taxa reported by Cruz-Motta et al. (2003) to be found in sediments below boulders during daytime low tides, only the cirratulid (Polychaeta) Cirriformia sp. and the sipunculid Phascolosoma sp. used the microhabitat below the boulder (Pattern 9; Table 5) consistently at any diel or tidal phase across all locations were they were observed (permanent habitat model).

Most of the temporal changes in individual taxa responded exclusively to the level of the tide (Patterns 1 to 6; Table 5, Fig. 5a-f). Few taxa, however, showed significant differences in relation to time of day (Pattern 7; Table 5, Fig. 5g) or due to interactions between time of day and level of tide (Pattern 8 and exceptions in Pattern 3; Table 5, Fig. 5h). These results strongly suggest a very dynamic migrational movement in response to the movement of the tides and, in a minor degree, to time of day. This has similarly been the case in studies of internal clocks determining the rhythms of intertidal organisms. The majority of these organisms respond exclusively to tides, and not to phase of the solar day (Palmer 1995, 2000). Some exceptions to this generalisation are the crabs Carnicus maenas (Warman et al. 1993) and Sesarma reticulatum (Palmer 1990) and the amphipod Orchestoidea tuberculata (Naylor \& Kennedy 2003).

The existence of several patterns of spatial distribution in relation to level of tides and, to a lesser degree, to the phase of the solar day, implies the existence of different mechanisms of migration. Migrational movements are considered to be different in different habitats. For example, it has been proposed that organisms living on rocky shores would tend to have 2 types of 'horizontal' movement: in and out of refuges as the phase of the day and or tide changes ('isospatial' strategy) or up- and downshore with the phase of the tide ('isophasic' strategy; e.g. Chelazzi et al. 1988). On the other hand, organisms living in sediments tend to show 'vertical' movement, emerging from the sediment into the water and moving back into the sediment according to the day and/or phase of tide (e.g. Warman et al. 1991, Takahashi \& Kawaguchi 1997). Note that the words 'horizontal' and 'vertical' have been stressed because they represent the main difference between the movements found in soft sediments versus hard substrata.

Boulder fields may comprise one of the few habitats in which there could be a combination of these strategies. In the present study, taxa such as the gammarid (Amphipoda) Hyale sp. and 2 unidentified isopods of the families Cirolanidae and Sphaeromatidae had patterns of spatial distribution (found in sediments away from boulders almost exclusively during low tide) that would be easily explained by the process of emergence typically seen in soft-bottom benthic organisms (e.g. De Ruyck et al. 1991, Yanicelli et al. 2001). Conversely, taxa like the anemone Actinia tenebrosa, which is typically found on hard substrata, had a pattern of spatial distribution that would be more easily explained by isospatial behaviour.

The patterns of spatial distribution of individual taxa described in this paper suggest, however, that there may be more mechanisms of migrational movements in intertidal boulder fields than those previously described for exclusively soft or hard substrata. For example, Patterns 1 and 2 (Table 5) may be the result of movements of organisms up and down the shore with the phase of the tide (isophasic), but this movement may result in taxa leaving or entering sediments below boulders, a potential refuge (Pattern 1; Table 5), while other taxa (Pattern 2; Table 5) do not.

In addition, due to the 3-dimensionality added by boulders, isophasic movements in relation to the phase of the tide by some organisms may be in 2 different dimensions, with some organisms moving up and down the shore horizontally, and others moving up and down the boulders vertically. An example of the later is the sea-urchin Helicon pruinosus, which migrates from the bottom to the top of boulders as the tide rises (Henninger \& Hodgson 2001). The presence of boulders may be important in determining and/or modulating the mechanisms of intertidal migration and the consequent patterns of spatial distribution for 2 reasons: (1) the added 3-dimensionality created by the boulders, and (2) because the boulders are potential refuges and, as proposed by some authors (e.g. Lawrie et al. 1999), migration of some taxa may depend on the availability of refuges. For example, some species may be intrinsically isospatial, but a shortage of refuges may force them to move isophasically.

The idea of refuge itself has to be considered cautiously. Some investigators have the intuitive idea that the micro-environment below a boulder is a refuge, whereas sediments next to it or the areas on the top of boulders are not (e.g. Rao \& Sarma 1979, Rocha 1995). It may be, however, that the top of a boulder constitutes a refuge for taxa whose predators arrive in sediments below boulders during high tide (e.g. the taxa in Patterns 3 \& 5 in Table 5). A similar process has been reported for the snail Littorina irrorata that moves up and down stalks of seagrass with the tide, and stays in a spatial refuge out of the reach of predators (e.g. Vaughn \& Fisher 1988). In these circumstances, the concept of isospatiality would have to be reconsidered.

The above are only a few of many possible types of movement that could exist and which could explain the patterns observed in this study. Fig. 6 presents 


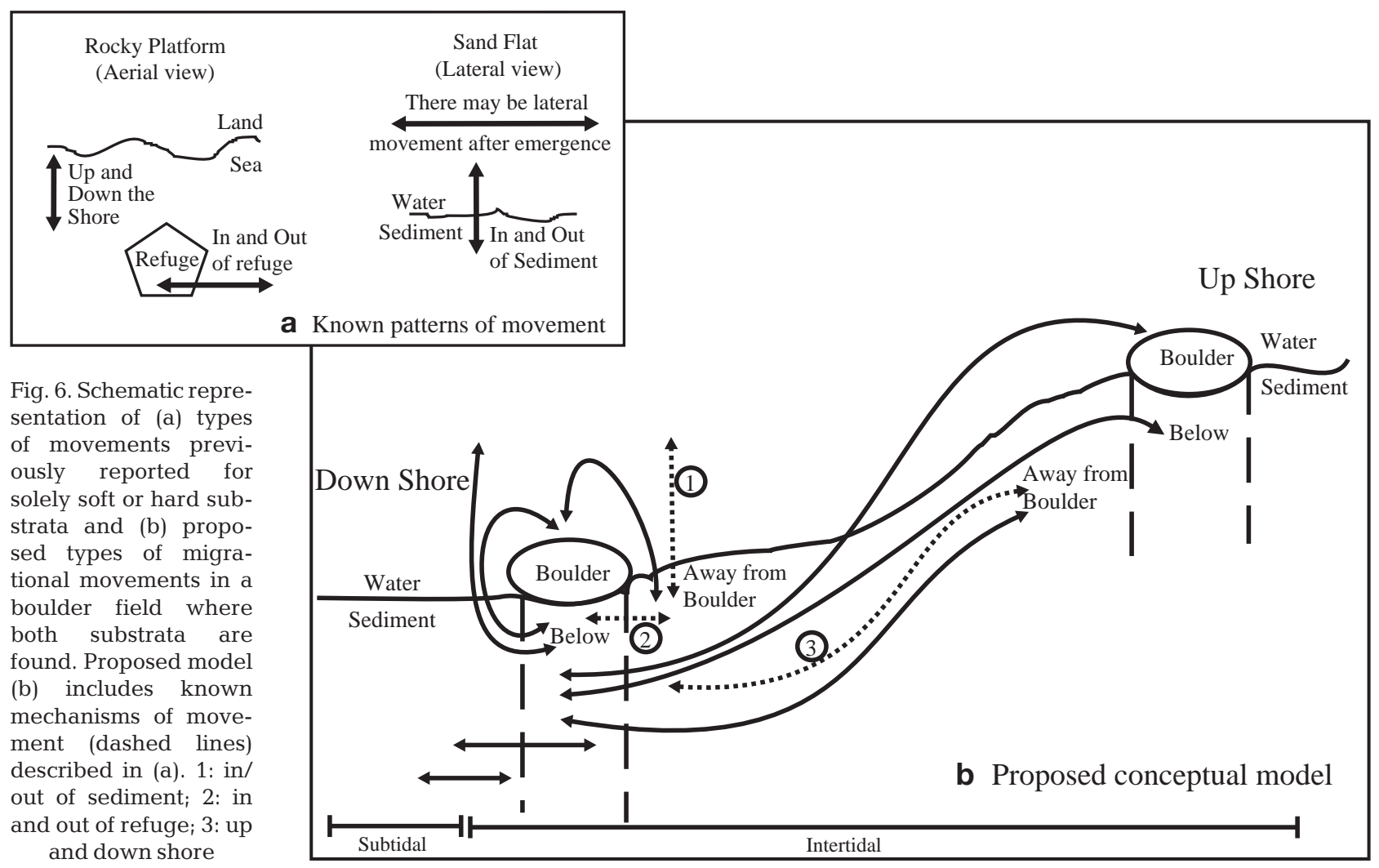

a schematic representation of the possible types of migrational movements that might be found in intertidal boulder fields, and compares them with the main mechanisms previously reported for intertidal sandflats and rocky shores. The situation would be more complicated if other possible pathways for assemblages living in other micro-environments associated with boulder fields (e.g. those on top of boulders), but not considered in this study, were included.

\section{CONCLUSIONS}

This study has shown that assemblages in sediments associated with boulder fields respond strongly to tidal and, to a lesser degree, diel fluctuations. Very few organisms used a specific micro-environment (sediments either below or away from boulders) as a permanent habitat. This study has also shown that more mechanisms could potentially explain migrational movements of organisms in boulder fields than those predicted by models proposed for either hard or soft substrata. Consequently, a new conceptual model on the possible migrational mechanisms is suggested. To evaluate this model, further studies are needed to test hypotheses on the different patterns of movements proposed herein.
Acknowledgements. I gratefully thank F. Barros, D. Blockley, F. Bulleri, S. Cummins, M. Cummins, G. Cruz, J. Cruz and T. Glasby for their assistance in the field, and A. Kazandjian for her assistance in the laboratory. Special thanks to M. G. Chapman, S. Cummins, T. Glasby, G. Palomo, F. Rossi, T. Tolhurst, A. J. Underwood and 3 anonymous reviewers for valuable improvements to the manuscript. Financial support was given by the Australian Research Council through the Centre for Research on Ecological Impacts of Coastal Cities and an International Postgraduate Research Scholarship at the University of Sydney.

\section{LITERATURE CITED}

Ameyaw-Akmufi CA, Naylor E (1987) Temporal patterns of shell gape in Mytilus edulis. Mar Biol 95:237-242

Armonies W (1992) Migratory rhythms of drifting juvenile mollusks in tidal waters of the Wadden Sea. Mar Ecol Prog Ser 83:197-206

Barnes DKA, Crook AC (2001) Quantifying behavioural determinants of the coastal European sea-urchin Paracentrotus lividus. Mar Biol 138:1205-1212

Blanchard GF, Simon-Bouhet B, Guarini JM (2002) Properties of the dynamics of intertidal microphytobenthic biomass. J Mar Biol Assoc UK 82:1027-1028

Cabral H, Costa MJ (2001) Abundance, feeding ecology and growth of 0-group sea bass, Dicentrachus labrax, within the nursery areas of the Tagus estuary. J Mar Biol Assoc UK 81:679-682

Chapman MG (2002) Early colonization of shallow subtidal boulders in two habitats. J Exp Mar Biol Ecol 275:95-116

Chelazzi G, Focardi S, Deneubourg JL (1988) Analysis of 
movement patterns and orientation mechanisms in intertidal chitons and gastropods. In: Chelazzi G, Vannini M (eds) Behavioral adaptation to intertidal life. NATO series Vol 151, Plenum Press. New York, p 173-184

Clarke KR (1993) Non-parametric multivariate analyses of changes in community structure. Aust J Ecol 18:117-143

Cruz-Motta JJ, Underwood AJ, Chapman GM, Rossi F (2003) Benthic assemblages in sediments associated with intertidal boulder-fields. J Exp Mar Biol Ecol 285:383-401

De Ruyck A, McLachlan A, Donn TE (1991) The activity of three intertidal sand beach isopods (Flabellifera: Cirolanidae). J Exp Mar Biol Ecol 146:163-180

Gevaert F, Creach A, Davoult D, Migne A, Levavasseur G, Arzel P, Holl AC, Lemoine Y (2003) Laminaria saccharina photosynthesis measured in situ: photoinhibition and xanthophyll cycle during a tidal cycle. Mar Ecol Prog Ser 247:43-50

Henninger TO, Hodgson (2001) Foraging activity of Helcion pruinosus (Patellogastropoda) on a South African boulder shore. J Molluscan Stud 67:59-68

Hicks GRF (1992) Tidal and diel fluctuations in abundance of meiobenthic copepods on an intertidal estuarine sandbar. Mar Ecol Prog Ser 87:15-21

Hutchings P (1999) Taxonomy of estuarine invertebrates in Australia. Aust J Ecol 24:381-394

Kim TW, Choe JC (2003) The effect of food availability on the semilunar courtship rhythm in the fiddler crab Uca lactea (de Haan) (Brachyura: Ocypodidae). Behav Ecol Sociobiol 54:210-217

Lawrie SM, Speirs DC, Raffaelli DG, Gurney WSC, Paterson DM, Ford R (1999) The swimming behaviour and distribution of Neomysis integer in relation to tidal flow. J Exp Mar Biol Ecol 242:95-106

McGuinness KA (1984) Communities of organisms on intertidal boulders: the effects of disturbance and other factors, $\mathrm{PhD}$ thesis, University of Sydney

McGuinness KA (2002) Of rowing boats, ocean liners and tests of the ANOVA homogeneity of variance assumption. Austral Ecol 27:681-688

Mendes S, Turrell W, Luetkebohle T, Thompson P (2002) Influence of the tidal cycle and a tidal intrusion front on the spatio-temporal distribution of coastal bottlenose dolphins. Mar Ecol Prog Ser 239:221-229

Naylor E (1988) Clock-controlled behaviour in intertidal animals. In: Chelazzi G, Vannini M (eds) Behavioural adaptation to intertidal life, NATO series Vol 151. Plenum Press. New York, p 125-136

Naylor E, Kennedy F (2003) Ontogeny of behavioural adaptations in beach crustaceans: some temporal considerations for integrated coastal zone management and conservation. Estuar Coast Shelf Sci 58S:169-175

Oliver L, Beattie AJ (1993) A possible method for the rapid assessment of biodiversity. Conserv Biol 7:562-568

Editorial responsibility: John Gray (Contributing Editor), Oslo, Norway
Palmer JD (1990) Daily and tidal components in the persistence activity rhythms of the crab Sasarma. Mar Behav Physiol 17:177-187

Palmer JD (1995) The biological rhythms and clocks of intertidal animals. Oxford University Press, New York

Palmer JD (2000) The clocks controlling the tide-associated rhythms of intertidal animals. BioEssays 22:32-37

Rao LM, Sarma DV (1979) Distribution, characters \& habits of Clypeomorus clypeomorus Jousseaume (Gastropoda: Cerithiidae) of the Visakhapatnam coast. Indian J Mar Sci 8:50-53

Rocha R (1995) Abundance and distribution of sessile invertebrates under intertidal boulders (Sao Paulo, Brazil). Bol Inst Oceanogr 43:71-88

Saigusa M (2001) Daily rhythms of emergence of small invertebrates inhabiting shallow subtidal zones: a comparative investigation at four locations in Japan. Ecol Res 16:1-28

Saigusa M, Kazushi O (2000) Emergence rhythms of subtidal small invertebrates in the subtropical sea: nocturnal patterns and variety in the synchrony with tidal and lunar cycles. Zool Sci 17:241-251

Short A (1993) Beaches of the New South Wales coast: a guide to their nature, characteristics, surf and safety. Australian Beach Safety and Management Program, Beaconsfield

Short A, Trenaman NL (1992) Wave climate of the Sydney region, an energetic and highly variable ocean wave regimen. Aust J Mar Freshw Res 43:765-791

Takahashi K, Kawaguchi K (1997) Diel and tidal migrations of the sand-burrowing mysids, Archaeomysis kokuboi, A. japonica and Iiella ohshimai, in Otsuchi Bay, northeastern Japan. Mar Ecol Prog Ser 148:95-107

Underwood AJ (1997) Experiments in ecology: their logical design and interpretation using analysis of variance. Cambridge University Press, Cambridge

Vaughn CC, Fisher FM (1988) Vertical migration as a refuge from predation in intertidal marsh snails: a field test. J Exp Mar Biol Ecol 123:163-176

Warman CG, O'Hare TJ, Naylor E (1991) Vertical swimming in wave-induced currents as a control mechanism of intertidal migration by a sand-beach isopod. Mar Biol 111: $49-54$

Warman CG, Reid DG, Naylor E (1993) Variation in the tidal migratory behaviour and rhythmic light-responsiveness in the shore crab, Carcinus maenas. J Mar Biol Assoc UK 73:355-364

Yamaguchi T (2001) Daytime larval release of the fiddler crab, Uca lactea (Decapoda, Brachyura, Ocypodidae). Crustaceana 74:545-555

Yannicelli B, Palacios R, Gimenez L (2001) Activity rhythms of two cirolanid isopods from an exposed microtidal sandy beach in Uruguay. Mar Biol 138:187-197

Submitted: June 15, 2004; Accepted: October 29, 2004

Proofs received from author(s): March 29, 2005 